\title{
The Evaluation System Model and Empirical Analysis of College Teacher Performance
}

\author{
Shang Hangbiao ${ }^{1, a}$, Qu Wenyu ${ }^{1, b}$, Tian Guoshuang ${ }^{1, c}$ \\ ${ }^{1}$ Northeast Forestry University, Harbin, 150040, China \\ aemail: shbszz@163.com, bemail:quwenyu@163.com, 'email:tgs1963@sina.com
}

Keywords: College Teachers; Performance Evaluation; Parameter Evaluation; System Analysis

\begin{abstract}
Based on the existing research, the establishment of evaluation index system of university teachers, on this basis, the method based on data envelopment sphere build college teachers performance assessment model. Then using kernel function instead of vector inner product, explore the dynamic evaluation model of university teachers' performance. Then teachers in colleges and universities to evaluate the parameters of the performance evaluation model and choice. Finally by a university technical economic teaching and research section 14 teachers performance indicators in 2011 points to verify the college teachers performance assessment model.
\end{abstract}

\section{Introduction}

College teacher performance evaluation is a dynamic complicated system [1], has the interactive relationship between characteristics of some uncertain factors and increased the difficulty of the evaluation, subjective factors should be considered in the evaluation of many factors, so the single use a kind of method is difficult to reflect the essence of teacher evaluation [2]. In order to make evaluation more university teachers' professional characteristics, use interactive relationship thinking, using system analysis method to study the decision making process, influencing factors in-depth analysis, establish evaluation system model[3]. Considering some of the factors are qualitative, can't completely rely on mathematical method, need to get the quantitative operation and qualitative analysis of organic together, make the evaluation more scientific, reasonable and comprehensive[4-8]. This paper will be combined with analytic hierarchy process (ahp), fuzzy analytic hierarchy process (ahp) and data envelopment analysis for three kinds of evaluation methods, and to integrate them, used for performance evaluation of higher school teachers.

\section{The construction of university teachers' performance evaluation model}

University teachers performance appraisal problems in pattern recognition can be classified into category classification problem [9]. There are several methods can be used to solve classification problems, such as the density estimation, boundary method and the reconstruction method, etc., of all the category classification problem, there are two fundamental issues to consider, the first basic question is the distance between state point calculations, or state points belong to the probability of the target class (and the similarity of the target class). The second basic question is the starting point of the distance or similarity (entrance) how to determine. If the new state point and the target class is less than the distance or similarity, the starting point of the distance or similarity, the new state point is accepted into the target class. In $\mathrm{d}(\mathrm{z})$ said state point, the distance to the target class $\mathrm{p}(\mathrm{z})$ said state points and the similarity of the target class, entry or starting point and $\operatorname{said}_{d} \theta_{d}$ and $\theta_{p}$, the state point to accept conditions can be expressed as the following form:

$$
f(z)=I\left(d(z)<\theta_{d}\right)
$$

Or with a state point and the similarity of the target class to indicate:

$f(z)=I\left(p(z)>\theta_{p}\right)$

$I($.$) is the indicator function:$ 


$$
I(A)= \begin{cases}1 & \text { if } \mathrm{A} \text { is true } \\ 2 & \text { otherwise }\end{cases}
$$

Assume that a data set including $\mathrm{N}$ data objects: $\left\{x_{i}, i=1, \ldots, N\right\}$. This is after a description of the data to describe the data, David multi-dimensional sphere with a minimum volume of all (or most) of the target data points used to describe the data set [9]. Describe the data set in this way there is a problem, that is, if the data is more discrete, the end result will be a huge sphere, making the entire model of the decline in the ability to identify the high sensitivity of the position of individual data objects, and a description of the data the effect is not good, so this article by introducing a slack variable, allowing some of the target state point is missing from the sphere.

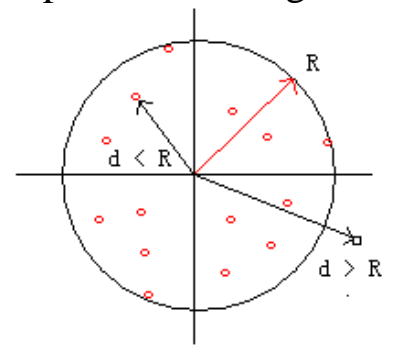

Normal state, the state of the super ball point and then minimize the radius of the sphere with A and radius $\mathrm{R}$ :

$$
F\left(R, a, \xi_{i}\right)=R^{2}+C \sum_{i} \xi_{i}
$$

Select the appropriate parameter $\mathrm{C}$ can strike a balance in the volume of a sphere and the error rate. (2) constraints and (1) the minimum:

$$
\left(x_{i}-a\right)^{T}\left(x_{i}-a\right) \leq R^{2}+\xi_{i} \quad \forall_{i}, \xi_{i} \geq 0
$$

(1) and (2) after the merger build the Lagrangian function:

$$
L\left(R, a, \alpha_{i}, \xi_{i}\right)=R^{2}+C \sum_{i} \xi_{i}-\sum_{i} \alpha_{i}\left\{R^{2}+\xi_{i}-\left(x_{i}^{2}-2 a x_{i}+a^{2}\right)\right\}-\sum_{i} \gamma_{i} \xi_{i}
$$

Which Lagrangian $\alpha_{i} \geq 0, \gamma_{i} \geq 0$, The set of partial differential is 0 , you can get new constraints:

$$
\begin{aligned}
& \sum_{i} x_{i}=1, \quad a=\frac{\sum_{i} \alpha_{i} x_{i}}{\sum_{i} \alpha_{i}}=\sum_{i} \alpha_{i} x_{i} \\
& C-\alpha_{i}-\gamma_{i}=0 \quad \forall_{i}
\end{aligned}
$$

Because $\alpha_{i} \geq 0$ and $\gamma_{i} \geq 0$, this paper use $0 \leq \alpha_{i} \leq C \forall_{i}$ Erasing the variables $\gamma_{i}$ from the constraint condition (4):

$$
L=\sum_{i} \alpha_{i}\left(x_{i} \cdot x_{i}\right)-\sum_{i, j} \alpha_{i} \alpha_{j}\left(x_{i} \cdot x_{j}\right)
$$

Constraint becomes:

$0 \leq \alpha_{i} \leq C ; \quad \sum_{i} \alpha_{i}=1$

(4) in the second equation shows that the center of the sphere is a linear combination of all data, the weighting factor in the data set, only a small part of the data to the formula (2) equality holds when equality holds,these data point in the sphere, this article also in the sphere of data points called support vectors. Be referred to as support vector data points, the coefficient $\alpha_{i}$ is not 0 , in describing the characteristics of the ball, only these support vector enough. The radius $\mathrm{R}$ of the sphere can be obtained by calculating the center of the sphere to the support vector distance, in general, support vector coefficients $\alpha_{1}$ are slightly smaller than parameter $\mathrm{C}$, if the data point of the coefficient is equal to $\mathrm{C}\left(\alpha_{i}=C\right)$, this data point will fall outside the ball, this data point, referred 
to herein as college teachers performance is not satisfactory shape (outlier) in the discussion later in this article will discuss in detail the parameters $\mathrm{C}$.

In order to determine whether a test data point is contained within the ball, the paper must first calculate this data point the distance to the center of the sphere, when the data points to the distance of the center of the sphere is smaller than the ball radius $\left((z-a)^{T}(z-a) \leq R^{2}\right)$. This article considers the data point is to be included within the sphere, which is a normal response to state point. The center of the sphere with the support vector that can be written as the following formula:

$(z \cdot z)-2 \sum_{i} \alpha_{i}\left(z \cdot x_{i}\right)+\sum_{i, j} \alpha_{i} \alpha_{j}\left(x_{i} \cdot x_{j}\right) \leq R^{2}$

When the data points fell within the sphere, the data points is the normal state, fell to the outside of the sphere, the point is that college teacher performance status point.

\section{College teacher performance evaluation model}

This paper all vector product kernel function $\left(K\left(x_{i} \cdot x_{j}\right)\right.$ ) instead of looking for the data envelopment described problem is transformed into the following form:

$$
L=\sum_{i} \alpha_{i} K\left(x_{i}, x_{i}\right)-\sum_{i, j} \alpha_{i} \alpha_{j} K\left(x_{i}, x_{j}\right)
$$

The constraint condition is: $0 \leq \alpha_{i} \leq C, \sum_{i} \alpha_{i}=1$ 。 The test data points are accepted when the following conditions are met:

$$
K(z, z)-2 \sum_{i} \alpha_{i} K\left(z, x_{i}\right)+\sum_{i, j} \alpha_{i} \alpha_{j} K\left(x_{i}, x_{j}\right) \leq R^{2}
$$

Different kernel function in the input space to the formation of different data describe the boundaries, the task now is to find a suitable kernel function $\left(K\left(x_{i} \cdot x_{j}\right)\right.$, the limited space here This article discusses two choices: polynomial kernel function and Gaussian kernel function.

Polynomial kernel function is actually an extension of the inner product: $\left(K\left(x_{i}, x_{j}\right)=\left(x_{i} \cdot x_{j}+1\right)^{d}\right)$, where the parameter $\mathrm{d}$ is a polynomial function of power (Vapnik, 1995). This kernel function vector inner product of the by seeking original vector space vector inner product and to obtain a higher latitude space image, For example, when $\mathrm{d} 2$, the two-dimensional space vector $\left(x_{1}, x_{2}\right)$ will be mapped to the five-dimensional space :

$\left(x_{1}, x_{2}, x_{1} x_{2}, x_{1}^{2}, x_{2}^{2}\right)$

In order to prevent the feature space is too large, we can using the Gaussian kernel:

$$
K_{G}\left(x_{i}, x_{j}\right)=\exp \left(-\left(x_{i}-x_{j}\right)^{2} / s^{2}\right)
$$

For the performance evaluation of university teachers would be better accepted principles into:

$-2 \sum_{i} \alpha_{i}^{2} K_{G}\left(z, x_{i}\right) \leq R^{2}-C_{x}-1$

Ten data points included in the two-dimensional feature space, we use a support vector based on the Gaussian envelope description (SVDD) to describe the scope of data points, wherein s ranging from very small to very large changes (1-25)This article can be noted from the figure as s increases, the number of support vectors in the reduced description of the function is closer to a sphere.

This article can be obtained by the exact solution, in two different extreme cases, s small value and s great value, and for very small $\mathrm{S},\left(K_{G}\left(x_{i}, x_{j}\right) \approx 0, i \neq j,\right)$ and $\left(\stackrel{L=1-\sum_{i} \alpha_{i}^{2}}{)}\right),\left(\alpha_{i}=1 / N\right), \mathrm{L}$ functions as a maximumvalue, its value is equal to $1-1 / \mathrm{N}$.

For very large values of $\mathrm{s},\left(K_{G}\left(x_{i}, x_{j}\right)=1\right)$ and $\left({ }^{L=1-\sum_{i} \alpha_{i}^{2}-\sum_{i+j} \alpha_{i} \alpha_{j}}\right)$, this time the conditions of the $\mathrm{L}$ function takes a maximum value that is: there is only one $\left({ }^{\alpha_{i}}=1\right)$, all the distance to the center of the sphere of 0 , the rightmost picture showing a the reality of the limitations of the data 
description is a minimum sphere of all data points, no college teachers performance ideal point.

\section{Conclusion}

This article draws on the interactive relationship between thinking, system analysis and evaluation method of university teachers performance evaluation. Proposed a new evaluation system model, and applied to the research on performance evaluation of university teachers in order to achieve a comprehensive evaluation of a teacher in an institution of higher learning performance. Specifically, the building of the college teachers performance evaluation system, including the evaluation index, evaluation model, the dynamic evaluation model and parameter selection. For College Teachers 'Performance Evaluation in the future, the need to further explore the mechanism and relationship of College Teachers' Performance Evaluation relational interaction interactive Higher Performance Evaluation [10], through breakthrough research paradigm, to promote the relationship between interactive science teacher performance evaluation practice and research applications [11].

\section{Acknowledgment}

This paper was funded by projects for Humanities and Social Sciences of Department of Education of Heilongjiang Province(12514026), and the Fundamental Research Funds for the Central Universities (DL11CC05).

\section{References}

[1] Wei-ying, SUN Rui College teacher performance appraisal to performance management transformation [J]. Economics and Management Research, 2006 (01): 56-57.

[2] Ting Dongxiang. A college teacher accountability and performance structural model reconstruction [J]. Higher Engineering Education Research, 2011 (05): 125-129.

[3] Peng Weiping. University Teachers' Performance Evaluation Preliminary Study [J]. Ningbo College, 2004 (03): 40-42.

[4] Li Changhua University Teachers' Performance Evaluation [J]. NAEA of 2007 (05): 91-95.

[5] Su Junxing, Yin Zhenji. Japanese university teacher evaluation system and Reference [J]. Dalian University of Technology, 2010 (05) :121-124.

[6] Tian Jing. Higher School Teacher Stages of Development and evaluation system [J] Journal of Education, 2006 (02) :58-61.

[7] Yu Qiangmei. University teachers wage levels 50 years longitudinal analysis [J]. Modern University Education, 2011 (04) :47-51.

[8] Wang Ruomei. Nearly a decade of domestic College Teaching Evaluation Research and Practice Review [J]. Jiangsu Higher Education, 2008 (03): 70-73.

[9] Li Ning. University Teachers' Performance Evaluation System Construction [J]. EDUCATIONAL RESEARCH, 2009 (3) :90-91.

[10]Jiang Xiangyang. Teacher Incentive mechanism design [J]. Reformation \& Strategy, 2004 (06) :71-74.

[11]He Baiyun. Teacher Incentive mechanism to build [J] People's Forum, 2010 (17): 206-207. 